\title{
The Application of Financial Engineering on the Management of Internet Financial Risks
}

\author{
Aijun Zuo, Jingwei Zhong \\ Nanchang Institute of Science\& Technology, Nanchang, Jiangxi, 330108
}

Keywords: Internet Finance; risk; financial engineering

\begin{abstract}
With the continuous development of Internet technology, the cooperation between the financial industry and Internet technology has become even closer. In the development of financial engineering, how to effectively integrate the traditional financial industry and Internet finance has become a problem that many people are researching and discussing. Because the Internet is developing too fast, there are no laws and regulations that impose certain restrictions and controls on internet finance. In addition to the openness of the Internet, there is also a certain risk of internet finance. Only a reasonable and effective control and management of such risks can make the Internet finance more comprehensively developed and thus improve the economic development of our country. Based on the concept of internet finance, this paper analyzes the problems existing in the development of internet financial risks under financial engineering, and then studies the management and prevention mechanisms of internet financial risks under financial engineering.
\end{abstract}

\section{Introduction}

With the arrival of the era of big data, many industries have undergone many changes, and even some changes have been earthshaking. In this era, if it does not change, it can only be eliminated by the times and become a dinosaur in the long history. The entire system of the financial industry is also constantly changing. The financial industry and Internet technology are mutually integrated and transformed from traditional operating models into innovative operating concepts. For China, the combined development of the Internet and finance is still in the stage of development. Many technologies are not mature and standardized, so it will bring financial risks to a certain extent. It is precisely this way that whether it can effectively manage and prevent such risks has become a problem that the financial industry is exploring, because it is also a necessary condition for the long-term development of Internet finance.

\section{Overview of Financial Engineering and Internet Finance}

At the end of the 20th century, the rapid development of corporate finances and various types of banking services in western developed countries led to the concept of financial engineering and the wider use of financial engineering. Some scholars believe that financial engineering involves the design, development, and effective application of innovations and methods in financial engineering, and that certain problems exist in the process of financial development. However, some scholars have divided financial engineering into broad and narrow senses. In a broad sense, financial engineering must take into account various aspects of the risks involved in dealing with customers, and manage and control this risk issue. Industry structured and powerful tools. On the other hand, the understanding of financial engineering should be more biased and technically speaking. For risk management tools and technologies, financial engineering is the key to management.

Internet finance is an innovative field created by the combination of the traditional financial industry and the Internet. Although Internet finance is a relatively new field, it still has the concept of rational thinking and financial theory in the traditional financial industry. The difference between internet finance and the traditional financial industry is different from the medium used by both. The purpose of internet finance is to make the traditional financial industry more transparent, reduce the intermediate costs and facilitate customers through the Internet and its advantages, 
increase customer engagement. In a simple sense, any Internet application that involves generalized finance can be called Internet finance.

\section{Risks in the Development of Internet Finance}

The Internet is a virtual network, because it is not a real, accessible object, so it will inevitably be accompanied by this certain risk problem. It is also the emergence of this problem, which has, to some extent, promoted a more stable network payment. development of. Of course, the risk does not mean that there will be security problems, but with the increasingly frequent network payments, this risk problem is also highly concerned by people. There may be a series of problems such as the out-of-control of internal management, the fraud of external payments on the Internet and the illegality of basic relationships.

Internet finance and the traditional financial industry have many differences, but both have their own advantages. Internet finance can use the advantages of the network and can complete basic financial services online. It can not only reduce costs, but also make the operation more efficient. The convenience is simple. Of course, all things are not perfect, and there are bound to be flaws in their merits. Although Internet finance is convenient and concise, its own authority can only be realized by using the customer's experience, so there is a certain degree of difficulty in improving the trust. On the other hand, Internet finance is also affected by virtual networks. It will give customers an unrealistic feeling that they do not want to be as authentic as traditional finance. In addition, customers may still be stuck in the traditional financial concept, so they will have certain network trust problems.

Internet finance can use financial services to the Internet, allowing customers to enjoy more convenient and better financial services. In fact, Internet finance has a better role in promoting the development of the modern financial industry, but the openness and extensiveness of the Internet also make this model a certain degree of risk. Internet finance is new to the traditional financial industry, and its changes are also relatively rapid. Therefore, the development of laws and regulations cannot keep up with policy changes. On the other hand, financial risks and network risks coexist. Emerging things inevitably have the necessity of its existence, but it is also because of emerging things that there are incomplete areas in many aspects, including the formulation of laws and regulations.

\section{The Financial Engineering under the Internet Financial Risk Prevention Mechanism}

Construct investor's risk defense system. At present, many investors' awareness of self-defense and safety awareness are relatively low. Therefore, they must promote and cultivate their awareness of risk protection and do their utmost to ensure that the initial defensive work is done as much as possible. With the aid of relevant regulations and legal knowledge, it is clear that online loans There are risks and hazards. It also strengthens the legal system that safeguards the rights and interests of customers. The legal system for protecting the rights and interests of customers is mainly for relatively normal products and services, but for Internet finance, it also has relatively special services and products, so the maintenance of the rights and interests of customers is not very comprehensive. In general, products are relatively numerous and there are also risks. Therefore, it is imperative to enhance the maintenance of customer interests, protection of personal information and reasonable trading rights.

There are still many flaws in the Internet financial management. At present, China does not have a perfect system. Therefore, the credit management system should be perfected. Based on relevant policy requirements, an Internet link should be established to further improve the security of the Internet. Similarly, in the Internet business, a unified system must be established so that all banks can communicate and appropriate information can be shared and learn from each other for common progress and development. Establish a common guarantee agency and industry association. At present, many systems in our country are not perfect, so the information obtained by banks may not be true, and the judgment results of banks on these data will also be biased, further increasing the 
risk of banks' funds. In addition, Internet finance has been developing and more problems may arise in the process. Therefore, the establishment of a comprehensive security system is an inevitable result, and it can better promote the further development of Internet finance.

Internet financial encryption. Under the traditional comparison of payment methods, Internet payment has a relatively large risk problem, because in the transaction process requires the use of a lot of data and more private information, and this information is very important, once the disclosure may be Cause serious consequences, so we must be rigorous in this aspect of encryption to ensure the security of the entire payment environment.

Internet financial authentication technology. When conducting transactions, it is necessary to ensure that the identities and information of both parties are accurate. Once an identity is not established, it will bring a lot of trouble, including of course the loss of its own property. Therefore, when the Internet authenticates its identity, it must be careful to ensure the accuracy of each identity, and video authentication is available when necessary. Secure electronic transaction protocol. In the electronic transaction process, the confidentiality technology of the password must be comprehensive and accurate. It can use a certain security protocol to control and restrict the transaction, so as to make the transaction more secure.

In the internet finance hacking prevention, it is even more necessary to intensify efforts. At present, during the normal use of the Internet, malicious attacks by some hackers will be used to steal important information, especially in the financial sector. The risks are even greater. Big. Therefore, in order to ensure the safe and effective implementation of Internet finance, you can build sophisticated anti-hacking systems against hackers, master skilled technology, and die from misfortune. You must be prepared to do well, and don't be overwhelmed after things happen. . The establishment of a more complete defense system can effectively reduce and avoid unnecessary losses.

When customers use the Internet to perform a series of operations on the Internet, the system must read personal information and record the behavior. In other words, there is a certain risk in the privacy of customers. Once privacy and personal information are exposed, it will cause huge losses to individuals. Therefore, we must establish a complete information privacy protection mechanism for the privacy issues with our customers. Only in this way can the customers use their products in peace of mind, but also allow more customers to participate in the use of the products, which will greatly help the development of Internet finance.

The development time of China's banks is relatively short, and there is a big gap with the management level of developed countries. Therefore, our country's bank management system should learn from the advantages of foreign countries and improve it on its own basis. The improvement of the bank's management system can effectively determine the emergence of risk escapades in internet finance, and it will be resolved when problems do not arise. Do a good job of bank management and build an architecture that adapts to the development of internet finance. For the management of Internet financial risks, we must establish a trade-off mechanism. We should not focus our rights on one person, balance the rights, make it appear in a state of disclosure, and establish a clear division of labor.

\section{Conclusion}

The development of network technology has led to the development of various industries, including the financial industry. The change of the financial industry from a traditional model to a completely new one also further promotes the development of technology. In the open network of Internet finance, there are various risk factors. Financial engineering has a good role in the development of Internet finance, which avoids many problems in operations and allows Internet finance to develop better. It also promoted the effective development of e-commerce. Making full use of financial engineering to strengthen management and increase the control and control of Internet financial risks can effectively promote the long-term development of the financial industry. 


\section{References}

[1] Wang Jing. On the use of financial engineering in financial risk management [J]. Modern Business, 2017(08):107-108.

[2] Yang Guogui. Analysis of the application of financial engineering in financial risk management [J]. Modern Economic Information, 2017(05):291-292.

[3] Wang Guanchao. On the current status of financial engineering development in China and its improvement measures [J]. Modern Business, 2017(03):100-101.

[4] Zhao Tianze. Application of Financial Engineering in Financial Risk Management [J]. Finance and Economics (Academic Edition), 2016(06):14. 